\title{
Análisis de las fuentes legislativas para el estudio de los derechos de la mujer en Chile
}

\author{
Rogelio Álvarez VicENTE \\ Académico de la Universidad Autónoma de Chile \\ Licenciado en Geografía e Historia por la Universidad de Salamanca. \\ Doctorando por la Universidad de Salamanca. Pasado y Presente de los Derechos Humanos.
}

Recibido: 28/02/2011

Aceptado: 04/04/2011

\section{RESUMEN}

Toda vez que los partidos políticos mantienen de facto en las sociedades modernas una enorme influencia en la actividad legislativa, es importante comprender su reacción programática y en la praxis frente a retos tan importantes como el que supone la aceptación de los Derechos Humanos $\mathrm{y}$, en concreto, su contribución al desarrollo normativo y práctico del paradigma de la igualdad de mujeres y hombres que, reconocido en principio y en teoría, es objeto de paradójicos recortes parciales, por acción u omisión.

Palabras clave: Derechos Humanos, visión de Género, Proyectos legislativos, Partidos Políticos

Analysis of legislative sources for the study of women's rights in Chile

\begin{abstract}
Considering the fact that political parties exert de facto in modern societies an enormous influence on legislative activity, it is important to understand their reaction not only programmed but also in practice when facing such major challenges as the one implied by the acceptance of Human Rights and, in particular, their contribution to policy and practical development of the paradigm of equality between women and men which, recognized in principle and in theory, is the subject of paradoxical partial cuts, by act or omission.
\end{abstract}

Key words: Human Rights, Gender viewpoint, legislative projects, Political Parties

\section{INTRODUCCIÓN}

Los partidos Demócrata Cristiano y Renovación Nacional de Chile ocupan el nicho de votantes de centro, el más numeroso, por lo que se encuentran en una posición privilegiada para influir en los legisladores y ciudadanos y para impulsar el desarrollo de políticas sociales acordes con el discurso internacional. Desde esta perspectiva habremos de ajustar explicaciones de sus olvidos y/o incumplimientos al respecto en materia de derechos de la mujer. Subrayamos el grado en que sus propuestas legislativas se ajustan o no al discurso normativo internacional, detectando la entidad de su aportación en las políticas económicas vigentes en el país, que condicionan su grado 
de cumplimiento. En la actualidad los Derechos Civiles y Políticos se encuentran completamente incorporados en los sistemas políticos de Occidente. No solo en las Constituciones, sino en el imaginario colectivo de las sociedades y Chile es un ejemplo de ello. En definitiva, se consideran ineludibles para alcanzar el bienestar humano. Sin embargo, son muchas las regiones del mundo, Chile entre ellas, que no otorgan la misma importancia a los Derechos Económicos, Sociales y Culturales, pese a recoger estos necesidades que todo ser humano debe tener cubiertas por el mero hecho de serlo.

\section{Derechos Humanos}

En la actualidad, con mucha frecuencia se recurre a los Derechos Humanos para argumentar un sin fin, a veces contradictorio (1), de intereses y necesidades (2). Es evidente la carga de autoridad que han conseguido, sobre todo a partir de la Declaración Universal de los Derechos Humanos de la ONU, en 1948. De esta manera, Derechos Humanos, derechos fundamentales, derechos universales, derechos culturales, ciudadanía.... son términos que están presentes en los discursos políticos, en los tratados internacionales, en las aulas, en los medios de comunicación, en las tertulias entre amigos entre otros. No obstante, enseguida notaremos que pese a tratarse de un concepto a priori conocido y aceptado por todos, las dificultades para su reconocimiento real y de facto son muchas (3).Y es que se trata de un concepto que influye directamente en mundos dispares tales como el judicial, el histórico, el sociológico, el religioso, el antropológico... que, a veces, difícilmente se compaginan.

\subsection{Origen de los Derechos Humanos}

La concepción de lo que hoy se conoce por Derechos Humanos corresponde a una etapa histórica relativamente reciente. Es propia de los tiempos modernos, es una idea que surge y se consolida a partir de, fundamentalmente, el siglo XVIII.La práctica de los derechos fundamentales nace precisamente cuando surge el Estado moderno, es decir, una forma política peculiar de organización de la comunidad política dotada de un poder político extraordinario: el reconocimiento de los Derechos Humanos es la respuesta jurídica a las amenazas planteadas por este nuevo poder político sin límites. Nacen para proteger al individuo frente a los peligros dimanantes de una entidad poderosísima (definida por Hobbes como "Leviatán"). Las primeras manifestaciones concretas de declaraciones de derechos individuales, con fuerza legal, fundadas sobre el reconocimiento de derechos inherentes al ser humano que el Estado está en el deber de respetar y proteger, las encontramos en las Revoluciones de Independencia Norteamericana (1776) e Iberoamericana (primer cuarto del siglo XIX), así como en la Revolución Francesa de 1789. Lo que en definitiva desencadenó la internacionalización de los Derechos Humanos fue la conmoción histórica de la Segunda Guerra Mundial y la creación de las Naciones Unidas. Surge así la Declaración de Derechos Humanos aprobada por las Naciones Unidas el 10 de diciembre de 1948. Los encargados de poner en marcha las nuevas instituciones supranacionales después de la Segunda Guerra Mundial entendían que todo orden socio-político —en este caso de carácter 
internacional-, se fundamenta en un código de valores, en unas normas de actuación y en una serie de instituciones propias que, al ser aceptadas por todos sus componentes, contribuyen a la estabilidad del sistema y por ende de la sociedad o comunidad en la que debe actuar. Para que esta estabilidad pueda lograrse y sea consistente, el nuevo orden que se pretende instaurar - y que nace, como el caso que nos ocupa, de una situación de paz que debe hacer posible la extensión y consolidación de los Derechos Humanos en los cinco continentes - debe ser lo más libre y justo posible.Los miembros de la comunidad deberían, de esta manera, aceptar esta concepción y tratarían de organizarse de común acuerdo a través de unas instituciones (por ejemplo la ONU) que generen, a su vez, unos principios básicos inalterables (los contenidos en la Carta fundacional de la ONU o la Declaración Universal de Derechos Humanos, entre otros), de obligado cumplimiento por todos. Y es que sólo en el siglo XX comenzaron a consolidarse y a extenderse los derechos sociales, cuya generalización llevó implícito el avance de la justicia social, caracterizado por una tendencia radical a la reducción de la desigualdad, que comenzó a percibirse, primeramente en la extensión de iguales derechos, nacidos directamente de la condición de miembros de la sociedad y que, por acuerdo social, está garantizada. A partir de ese momento, se hizo imparable, la lucha contra las desigualdades que frenan la plena participación ciudadana por medios sociales, políticos o económicos. Así, la conquista de los derechos sociales suponía una igualdad de estatus que compensaba las disparidades de ingreso y clase".

\subsection{Fundamentos de los Derechos Humanos}

Los Derechos Humanos son, en la actualidad, invocados constantemente; si bien, no existe similar preocupación social por justificarlos. Se da por supuesta su validez... pero bajo el aparente consenso occidental sobre la indiscutibilidad de los Derechos Humanos se oculta una divergencia profunda sobre su razón de ser, lo cual puede hacernos pensar que estamos hablando de un concepto poco fundado. La búsqueda de una fundamentación capaz de proporcionar una base moral para los Derechos Humanos fortalecerá el reconocimiento político - jurídico de los derechos. De hecho, múltiples fundamentos (iusnaturalismo, iuspositivismo fundamentos axiológicos, fundamentación consensual, utilitarismo etc...)(4) de los que disponemos en la actualidad son suficientes para muchas personas (incluyendo políticos, activistas de Derechos Humanos etc.) y favorecen su "normativación;" de la misma manera puede afirmarse que la discusión sobre la fundamentación de los Derechos Humanos estimula la conciencia moral (5) de sectores sociales diversos para intentar solucionar la situación de lo más desfavorecidos y mantener todo lo relacionado con la dignidad del hombre como tema central de las agendas políticas. Sin embargo, es innegable que la actuación de muchos Estados demuestra la fragilidad de los diferentes acuerdos en defensa de los Derechos Humanos, lo que indica la conveniencia de seguir buscando argumentos a su favor. 


\subsection{Concepto de los Derechos Humanos}

Es necesario destacar que no todo discurso igualitario o humanista es identificable sin más con el discurso de los Derechos Humanos y es posible una delimitación conceptual en consonancia con los trabajos realizados a tal fin por la ONU, referente de primera línea en nuestra investigación. En el preámbulo de la Declaración de Derechos Humanos de la ONU, aprobada por la Asamblea en 1948 se define a los Derechos Humanos como "facultades iguales e inalienables de todos los miembros de la familia humana, que estipulan la dignidad y el valor de la persona humana así como la igualdad de derechos de todos los seres humanos". Es necesario destacar que se entiende por persona humana al ser racional con capacidad moral (conciencia para diferenciar el bien del mal) para actuar.

\subsection{Caracteres esenciales de los Derechos Humanos}

Reconocer los Derechos Humanos supone aceptar la existencia de atributos inherentes a la persona, que no son una concesión de la sociedad ni dependen del reconocimiento de un Gobierno. Todo ello supone adentrarnos en el complejo mundo de los caracteres de los Derechos Humanos.

\subsubsection{Universalidad}

Por ser inherentes a la condición humana, todas las personas son titulares de los Derechos Humanos y no pueden invocarse diferencias de regímenes políticos, sociales o culturales (6) como pretexto para ofenderlos o menoscabarlos.

\subsubsection{Inalienables}

Los Derechos Humanos pertenecen en forma indisoluble a la esencia misma del ser humano; no pueden ni deben separarse de la persona y, en tal virtud, no pueden trasmitirse o renunciar a los mismos, bajo ningún título (7).

\subsubsection{Absolutos}

Los positivistas, en el contexto de la Ilustración, pretendieron otorgarles a los Derechos Humanos el carácter de absolutos, como lo demuestra la Declaración francesa de 1789. Irónicamente aplicaban el concepto iusnaturalista de que por ser naturales, los derechos humanos son absolutos. En la actualidad, el carácter absoluto de los Derechos Humanos encuentra difícil acogida toda vez que múltiples Constituciones de Estados democráticos y la propia declaración de Derechos Humanos de la ONU admiten cláusulas limitadoras o suspensivas. 


\subsection{Clasificación de los Derechos Humanos}

La clasificación de los Derechos Humanos puede hacerse siguiendo ciertas convenciones de los especialistas que, solo aparentemente, se basan en un enfoque historicista, tomando en cuenta la protección progresiva de los Derechos Humanos (8). La clasificación más conocida es la que distingue las llamadas tres "generaciones", a la que en los últimos tiempos se tiende a añadir una cuarta y una quinta.

\subsubsection{La Primera Generación de Derechos}

La "primera generación" de Derechos surge con las primeras liberales y en Europa cristaliza con la Revolución Francesa, en reacción contra el absolutismo. Se encuentra integrada por los denominados derechos civiles y políticos. Imponen al Estado el respeto de ciertos derechos (a la vida, a la libertad, a la igualdad, a la propiedad etc.).Por eso se les denomina derechos "negativos" o de abstención (9).

\subsubsection{Los Derechos de Segunda Generación}

Los derechos de "segunda generación están constituidos por los Derechos, que algunos consideran de tipo individual o subjetivos y otros denominan colectivos, los Sociales, Económicos y Culturales (10). Surgen como resultado de la Revolución Industrial, a partir de una tradición de pensamiento humanista y socialista; son de naturaleza económica y social, e inciden sobre la expresión de igualdad de los individuos. A fines del siglo XIX y principios del XX, debido al auge del movimiento obrero y a la aparición de partidos de ideología socialista, se empieza a calificar a los derechos civiles como meras libertades formales, si no se garantizan, a su vez, este tipo de derechos.

\subsubsection{Los Derechos de Tercera Generación}

En la categoría de los Derechos de Tercera Generación entran los Derechos de los pueblos o de Solidaridad (11), que se manifiestan a partir de la década de los años sesenta del siglo XX. En el año 1966, las Naciones Unidas mencionan en sus Pactos Internacionales los primeros derechos colectivos: El "derecho al desarrollo" y "derecho a la libre determinación de los Pueblos". Esta vez, su motor impulsor serán las reivindicaciones de determinados colectivos que reclaman legítimos derechos. Se trata de incentivar el progreso social y elevar el nivel de vida de todos los pueblos, en un marco de respeto y colaboración mutua entre las distintas naciones de la comunidad internacional. Gracias a ello se ha desarrollado el concepto de diálogo Norte - Sur, el respeto y la conservación de la diversidad cultural, la protección del medio ambiente, la conservación del patrimonio cultural de la humanidad etc. 


\subsubsection{Los Derechos de Cuarta Generación}

En los albores del siglo XXI, el desarrollo de las nuevas tecnologías (12) de la información vinculadas a la revolución de las telecomunicaciones ha planteado una nueva problemática. Las denominadas TIC (Tecnologías de la Información y Comunicación) han revolucionado tan intensamente nuestras relaciones sociales como en su momento la Revolución Industrial. La existencia de oportunidades, pero también de amenazas, en esta nueva sociedad hace perentorio la necesidad de crear garantías normativas y poner en marcha políticas públicas.

En definitiva, los derechos de cuarta generación se sostienen en la necesidad novedosa de conseguir que todos los individuos accedan a las tecnologías de información y comunicación, asegurar el flujo de intercambio de información mediante la transparencia de conocimientos y estimular la innovación y formación de capital humano.

\subsubsection{Los Derechos de Quinta Generación}

Inmersos en el mundo de la Globalización, las estructuras políticas, económicas y sociales evolucionan constantemente; lo mismo sucede con las necesidades de las personas. De esta manera se toma conciencia de la importancia que el deporte tiene para el ser humano. Se entenderá por deporte todo tipo de actividades físicas que, mediante una participación, organizada o de otro tipo, tengan por finalidad la expresión o la mejora de la condición física y psíquica, el desarrollo de las relaciones sociales o el logro de resultados en competiciones de todos los niveles.

\section{SITUACIÓN DE LA MUJER EN CHILE}

Los datos presentados en este apartado tienen principalmente como base el último Censo realizado en Chile, el de 2002. No obstante, numerosas instituciones han publicado proyecciones y estimaciones de fechas muy recientes, permitiéndonos así una actualización de los datos (13). Es conveniente subrayar que la "perspectiva de género" empleada en el análisis que se realiza a lo largo de toda la investigación distingue sexo de género. La palabra "sexo" hace referencia a la descripción de la diferencia biológica entre hombres y mujeres y no determina necesariamente los comportamientos sociales. El concepto "género" designa el papel o entidad que en cada sociedad se atribuye a cada uno de los sexos, es decir, se refiere a la construcción social del hecho de ser mujer y hombre, a la interrelación entre ambos y a las diferentes relaciones de poder/subordinación en que estas interrelaciones se presentan. En una palabra, los sistemas "Sexo-Género" son los conjuntos de prácticas, símbolos, representaciones, normas y valores sociales que las sociedades elaboran a partir de las diferencias sexuales. Este sistema ha determinado tanto la posición social diferenciada para mujeres y hombres como las relaciones desiguales entre ambos. El Sistema Sexo-Género históricamente ha generado una situación de discriminación y marginación de las 
mujeres en los aspectos económicos, políticos, sociales y culturales, así como en los ámbitos público y privado, estableciendo muy clara y diferencialmente la intervención de los hombres en la esfera productiva y de las mujeres a la reproductiva.La posición social diferenciada se construye a partir de la asignación de papeles, espacios, características e identidades diferentes para cada persona en razón a su sexo por lo que la situación puede modificarse. Sin duda las desigualdades existentes entre las condiciones de vida del hombre y de la mujer son determinantes a la hora de analizar la situación de esta última en la sociedad occidental en general y chilena en particular, lo cual supone una clara violación de la Convención sobre Eliminación de todas las Formas de Discriminación hacia las Mujeres (CEDAW), suscrito y ratificado por el Estado Chileno en el año 1989.Con la llegada de la democracia en 1990 van a mejorar ostensiblemente las condiciones de vida de los chilenos y chilenas. No obstante, toda vez que han pasado ya veinte años desde que dicho proceso se inició no ha lugar a medir los avances en comparación con el parámetro que supone el periodo anterior, sino con los estándares internacionales de Derechos Humano, en particular los de las mujeres, aplicables en un contexto democrático. Miles de personas viven en la actualidad en condiciones de extrema pobreza, un importante número de ellas son mujeres. Durante el último decenio, el número de mujeres pobres en el país ha disminuido; sin embargo, esta situación es particularmente grave entre las mujeres rurales y las jefas de hogar puesto que la precariedad de sus ingresos las sitúa en un riesgo mayor. En las últimas décadas, la tendencia general se ha dirigido hacia una mayor inserción laboral de las mujeres y la mejora de la calidad de los empleos. Las mujeres pobres tienen menos oportunidades económicas y menos autonomía que los hombres pobres. Su acceso a los recursos económicos, al capital, a la educación y capacitación y a los servicios de apoyo es más limitado. Su participación en la adopción de decisiones es insignificante por lo que es relevante conocer el impacto diferenciado de la pobreza entre hombres y mujeres y así concebir políticas y programas cada vez más eficaces y participativos. Sin embargo, es necesario subrayar que en Chile el trabajo femenino no es una opción, sino una obligación, si se quiere salir de la condición de pobreza.Incluso, cabe añadir, que la clase media tampoco puede prescindir del sueldo que aporta la mujer si se piensa en los gastos adicionales en que deben incurrir las familias ante las claras insuficiencias del sistema público en las áreas de educación y salud(14). Paradójicamente la discriminación de la mujer en el ámbito laboral persiste, como lo demuestra la tasa de actividad, la tasa de desempleo, las remuneraciones, la situación contractual, la previsión social y los índices de acoso sexual en el trabajo. La participación en pie de la igualdad de la mujer en la adopción de decisiones es una exigencia básica de justicia social o de democracia. Dicha participación permite lograr un equilibrio que refleje más fielmente la composición de la sociedad. Pese a que en la mayoría de los países hay una tendencia generalizada a la democratización, sigue habiendo una notable ausencia de mujeres en casi todos los niveles de la estructura gubernamental. De esta manera, al estar ausentes o escasamente representadas en los liderazgos políticos y económicos, no son consideradas actores sociales ni sujetos políticos relevantes. 
Es necesario resaltar la relación existente entre la escasa presencia femenina en los ámbitos legislativos y gubernamentales con la discriminación que viven las mujeres en los partidos políticos (15). Pese a los avances que en materia de salud sexual y reproductiva presenta Chile, queda mucho por hacer. Los derechos sexuales y reproductivos, en Chile, no son derechos reconocidos a nivel institucional o legal.

El Comité CEDAW muestra especial preocupación por la imposibilidad legal de practicar cualquier tipo de aborto en Chile, toda vez que afectan a la salud de la mujer, dan lugar a que aumente la mortalidad derivada de la maternidad y ocasionan nuevos sufrimientos cuando las mujeres son encarceladas por violar esas disposiciones. En el Parlamento, mientras tanto, se han presentado diferentes iniciativas de ley que han buscado aumentar las penas para las mujeres y los terceros que realizan abortos. Sin embargo, pese al carácter conservador de los miembros de las Cámaras, es palpable la crecida de una corriente que tiene más en cuenta los derechos de la mujer y que, bien sea por convicción personal o presión social, está en consonancia con los tratados internacionales que recomiendan no encarcelar a las mujeres que abortan.

Respecto a la situación de la mujer con respecto a su acceso a la salud, el $82 \%$ de la población femenina pertenece a FONASA, el sistema de salud público chileno, de peor cobertura que las ISAPRES (sistema de salud privado): Queda en evidencia la incompetencia del mercado para asegurar bienestar y equidad en salud a la población y en particular a la mujer. El Estado está realizando un esfuerzo enorme con el llamado "Plan Auge", que supone la creación de nuevos organismos con el fin de mejorar los servicios sanitarios en todo el país, instituciones, de origen político que vigilan tanto al sector privado como al público y establece la prioridad en su tratamiento de una serie de patologías, si bien existen una enorme variedad de enfermedades (16) que afectan principalmente a las mujeres que se encuentran fuera del Plan.

Una de las más graves violaciones de los Derechos Humanos de la mujer es la violencia (17) que se ejerce contra ellas y que se encuentra presente en todas las sociedades en mayor o menor grado (ya sea física, sexual y psicológica); dándose tanto entre las clases acomodadas como en las humildes, entre las personas cultas o entre aquellas con escasa preparación. En definitiva, son muchas las chilenas sometidas al acoso laboral, la violencia doméstica, la violación o incluso el asesinato por el mero hecho de ser mujeres. Las prácticas culturales perpetúan la situación de inferioridad de la mujer y contribuyen a que la violencia citada sea posible. Algunos grupos de mujeres, como las emigrantes, prostitutas, las que forman parte de minorías étnicas entre otras, deben ser objeto de atención especial pues son particularmente vulnerables a la agresión. Más de la mitad de las mujeres son víctimas de algún tipo de violencia en la región metropolitana (urbana y con más de la mitad de la población del país). Pero en una zona rural como la región IX (La Araucanía), el 47\% de la población femenina corre la misma suerte. El número de femicidios, como la forma extrema del control de las mujeres por parte de los hombres, es alto. Hasta el 13 de diciembre de 2010 ha habido 55 femicidios (18) apareciendo los esfuerzos gubernamentales por disminuirlos como insuficientes. Y es que se toman medidas legislativas en defensa de la mujer pero se sigue sin tratar la violencia contra la mujer como un fenómeno nacido en el seno de unos parámetros culturales machistas que sitúan a la mujer en una situación de 
desigualdad respecto al hombre y que fomentan, incluso, la misoginia al contemplar a la mujer como "inútil" para ciertos trabajos tradicionalmente masculinos y, por lo mismo, recluida a sus actividades domésticas, reproductivas y crianza de los hijos. El aumento del acceso de la población a la educación ha beneficiado de manera particular a la mujer, que alcanza niveles educativos similares o incluso superiores a los de los hombres. La importancia que adquiere la educación en un mundo tecnificado como el nuestro, va más allá del enriquecimiento cultural de la persona pues supone un requisito básico para conseguir una mayor competitividad y crecimiento económico; aspectos que deben conseguirse bajo el prisma de la equidad y mejora de la calidad de vida de todas las personas, hombres y mujeres. Pese al capital educativo acumulado por la mujer, éste no ha supuesto una participación económica, política y de poder en igualdad de condiciones con el hombre. En definitiva, la desigualdad de la mujer respecto al hombre persiste, pues todavía se mantiene la discriminación, originada en las costumbres, las normas sociales e incluso en la educación formal sexista. Respecto a los pueblos originarios, en Chile los indígenas se enfrentan a muchas situaciones que vulneran sus derechos, ya sea discriminación, negación cultural, marginalización económica y hoy persecución política. Pero es especialmente preocupante la situación de las mujeres y niñas indígenas".

Por ello, no podemos dejar de mencionar la reflexión que realiza D. José Saramago: "Yo creo que la emergencia de los pueblos indígenas - una emergencia que los haga salir de lo exótico, de lo folklórico- es una necesidad vital de esta América" (19).

\section{FUENTES LEGISLATIVAS}

Los proyectos de ley pertinentes al artículo que presentamos pretenden cambios que se consideran necesarios en lo relativo a la perspectiva de género concebida por los Principios del Pacto de Derechos Económicos, Sociales y Culturales y suponen una radiografía fiel de la visión de género mantenida por los parlamentarios.

\subsection{Derechos sexuales y reproductivos de las mujeres}

\subsubsection{Proyecto de Ley que "Tipifica y Sanciona el Acoso Sexual", Congreso Nacional de Chile, 24 de Octubre de 1994, Boletín del Congreso Nacional de Chile $\mathbf{N}^{\circ}$ 1419-07. Historial de la Ley: Ley $\mathbf{N}^{\circ}$ 20.005. Decreto. Oficial. 18 de Marzo de 2005. \\ El Proyecto se convirtió en Ley de la República, pero la intervención de muchos parlamentarios destacó que no era suficiente con legislar y que resultaba perentorio que todos los chilenos aceptaran la perspectiva de género para conseguir el reforzamiento efectivo de la dignidad de las mujeres. Conviene recordar que si bien las mujeres comparten cada vez más el papel de productoras, los hombres no asumen una redistribución de tareas domésticas acorde con el principio de corresponsabilidad de las mismas lo que implica una sobrecarga del esfuerzo de las mujeres e impide la conciliación de la vida laboral y familiar, de mujeres y hombres.En definitiva, de nada}


sirven leyes que protejan los derechos laborales y salariales de la mujer, si el hombre no asume las consecuencias de las transformaciones que se vienen dando en todos los aspectos en las sociedades occidentales actuales en general y chilena en particular.

\subsubsection{Proyecto de Ley que "Establece Ley Marco sobre Derechos Sexuales y Reproductivos. Congreso Nacional de Chile, jueves 19 de octubre de 2000. Boletín del Congreso Nacional ${ }^{\circ}$ 2608-11. Historial: Primer trámite constitucional. Sin urgencia.19 de octubre de 2000.}

Pese al carácter moderado del Proyecto de Ley que estamos analizando, no parece probable que supere los diferentes trámites parlamentarios dejando en evidencia el pensamiento conservador de la mayoría de los parlamentarios chilenos. No obstante, los legisladores que presentaron la moción para aumentar los derechos y libertades sexuales y reproductivos en Chile son conscientes del revulsivo que supone en su país y defienden su autoría, por lo que podemos inferir que poco a poco van apareciendo cambios en las mentalidades respecto a temas axiológicos, hasta ahora considerados "indefendibles" en Chile.

\subsubsection{Proyecto de Ley que "Introduce un nuevo tipo delictivo en el Código Penal, relacionado con los diagnósticos prenatales. Congreso Nacional de Chile, 14 de Enero de 2004. Boletín del Congreso Nacional N’3449-11. Historial: Archivado. 6 de Marzo de 2007.}

En definitiva, el Proyecto que "Introduce un nuevo tipo delictivo en el Código Penal, relacionado con los diagnósticos prenatales" supone un paso más en una concepción de género regresiva o discriminatoria de las mujeres pues pretende afinar en la prohibición de toda acción cuyo fin sea provocar un aborto incluidos ciertos avances que la técnica ha experimentado en las últimas décadas como los llamados "diagnósticos prenatales" que consisten en exámenes realizados "in útero" para detectar en el embrión o en el feto una enfermedad especialmente grave, ya que esta información puede decidir a muchos padres a optar por un aborto ante las dificultades físicas del nuevo ser, psicológicas para la familia y su entorno y, por supuesto, económicas. El seis de marzo de 2007 se solicita el archivo de la mención por haber transcurrido más de dos años sin que la Comisión se haya pronunciado sobre esta iniciativa legal (artículo 17 del Reglamento de la Corporación). Este rechazo no responde a una preocupación progresista, contra lo que pudiera parecer. En efecto, en Chile existe un seguimiento prenatal muy completo a todos los niveles, incluidas las personas sin posibilidades económicas y que, por lo tanto, pertenecen al sistema público de salud. El seguimiento del desarrollo del embrión y del feto, la utilización de las últimas tecnologías y el asesoramiento de profesionales está garantizado por Ley. La Iglesia Católica muestra periódicamente su preocupación en la prensa (20) por todo aquello que signifique un atentado contra la vida del "nasciturus", rechazando el aborto terapéutico, el eugenésico y toda intervención que busque la eliminación de la vida en gestación, pensando igualmente en las heridas de todo tipo que debe soportar la mujer que ha realizado un aborto. De esta manera toda madre conoce las condiciones de la criatura en gestación y por lo tanto se hace realmente difícil clasificar de delictiva la acción de los médicos, dada la obligación que 
tiene todo facultativo de denunciar cualquier tipo de acción tendente al aborto y que, en principio, la información pre - natal no pretende sino mejorar la calidad de vida de la madre y del "nasciturus". Los autores del Proyecto de Ley que "Introduce un nuevo tipo delictivo en el Código Penal, relacionado con los diagnósticos prenatales" pertenecen justamente a los partidos que estamos analizando en esta investigación y son coherentes con el programa de los partidos que representan.

\subsubsection{Proyecto de Ley que "Modifica disposiciones sobre prostitución". Congreso nacional de Chile, 7 de diciembre de 2006. Boletín del Congreso nacional $\mathbf{N}^{\circ}$ 4715-11. Historial: Primer trámite constitucional. Sin urgencia. 7 de diciembre de 2006.}

El proyecto que "Modifica disposiciones sobre protección" y que pretende modificar el artículo 367 del Código Penal (21) es propuesto por ocho diputados y diputadas. Todos pertenecen a la izquierda chilena salvo uno que es democratacristiano. No hay ningún diputado de Renovación Nacional. Los diputados y diputadas al presentar el citado Proyecto pretenden disminuir el trabajo sexual o prostitución callejera subrayando en primer lugar su preocupación por los vecinos de los sectores colindantes y en segundo lugar proteger a las propias personas que ejercen el comercio sexual en lo que respecta a su salud o a la delincuencia. De esta manera pretenden aumentar las penas para el proxeneta, máxime cuando utilizara violencia o tuviera algún grado de parentesco con la víctima. También se propone un aumento de las penas para la víctima. Pese a que el Proyecto no se muestra radical en ninguno de sus contenidos, hiere sensibilidades puesto que el tema de la prostitución, como otros muchos, no presenta interés para una clase política embebida de conceptos axiológicos que, al tiempo, los vulnera cuando lo cree oportuno. Se trata de una realidad que, según estos postulados, es preferible esconder. Dada la fuerza de "Alianza por Chile, podemos presumir que el proyecto, que inició su andadura en 2006 no va a tener acogida en ninguna de ambas cámaras y en la actualidad ni siquiera ha entrado a debate, pese a haber pasado más de cuatro años desde su presentación.

3.1.5. Proyecto de Ley que "Modifica el Código Penal y el Decreto Ley $\mathbf{N}^{\circ}$ 321, de 1925, para sancionar el "femicidio", y aumentar las penas aplicables a este delito". Congreso Nacional de Chile, 3 de abril de 2007. Boletín del Congreso Nacional $\mathbf{N}^{\circ}$ 4937 - 18. Historial: Ley de la República en octubre de 2010.

Los autores de la moción subrayan que la violencia contra la mujer aumenta con el paso de los años, tiene enorme conmoción pública, genera inseguridad y, por encima de todo, supone una de las más graves vulneraciones de la dignidad de la mujer. Por todo ello, la violencia de género en general y el "femicidio" en particular son fenómenos absolutamente reprochables. Es de destacar que en el Proyecto se pretende incorporar, conceptualmente, el tipo de femicidio, "como todo asesinato en que la víctima sea la cónyuge, conviviente o cualquier mujer con la que el agresor está o haya estado ligado por alguna relación afectiva" (22).Los políticos conservadores relacionan el femicidio más bien con la pobreza, la desestructuración de la familia o a fallos del sistema educativo. Fijada así la etiología de los delitos sexuales, los remedios buscados no irán 
dirigidos a promover la igualdad de varones y mujeres y proporcionar a las segundas autonomía, sino a reforzar la familia tradicional y a favorecer la reconciliación de sus miembros en caso de conflicto. Debemos destacar que los derechos sexuales y reproductivos de la mujer, que en los últimos tiempos van encontrando acogida en el discurso internacional, no encuentran demasiado eco en Chile y ni siquiera figuran en la agenda de debate de los parlamentarios. En definitiva, se genera un quiebre entre el pensamiento de los parlamentarios y el sentir de la población. En efecto, son muy numerosas las asociaciones, como la Red Chilena Contra la Violencia Doméstica y Sexual, que anualmente realizan campañas publicitarias denunciando el fenómeno (Slogan de 2007: "¡Cuidado!, el machismo mata"), realizando memoriales, debates, actos simbólicos y exhortando a las autoridades a concebir políticas coherentes y coordinadas y a centrarse no solo en asistir a las mujeres maltratadas sino también en promover condiciones que hagan posible que la mujer refuerce su condición de sujeto con poder de decisión sobre su vida (23). Es necesario acabar con un fenómeno que bebe en conceptos de vida machistas y de prepotencia masculina y que hoy, más que nunca, carecen de todo sentido. La mujer exige que se le reconozca como persona con derechos y en igualdad de condiciones y capacidades respecto al hombre. Sorprende que la Presidenta Bachelet no impulsara esta propuesta, como otras que defienden a la mujer, pese a su condición de fémina y de médico. No obstante, el Proyecto ha adquirido rango de Ley de la República el 13 de diciembre de 2010, ya siendo presidente el conservador Sebastián Piñera.

3.1.6. Proyecto de Ley que "Extiende a los padres trabajadores el derecho de alimentar a sus hijos y perfecciona normas sobre protección de la maternidad". Congreso Nacional de Chile, 3 de Abril de 2007. Boletín del Congreso Nacional N 4930-18. Historial: Primer trámite constitucional. Sin urgencia. 7 de Mayo de 2009.

En la moción que "Extiende a los padres trabajadores el derecho de alimentar a sus hijos y perfeccionar normas sobre protección de la maternidad" se destaca el enorme desarrollo que han tenido en los últimos años las normas sobre protección de la maternidad. Últimamente se ha buscado perfeccionar y extender los derechos de las madres, eliminar la discriminación para garantizar la igualdad de género e incorporar el reconocimiento de derechos y deberes de este colectivo para con sus hijos. El desarrollo en igualdad de los derechos de paternidad es una de los mayores retos para los defensores de los derechos de la mujer, toda vez que resolverán los efectos colaterales indeseados del reconocimiento de los derechos de maternidad dejando sin argumentos a los que afirman que contratar a una mujer es demasiado costoso. El Proyecto que nos ocupa sin duda se dirige en la dirección correcta pues incide en la importancia de que el padre se involucre en la crianza de sus hijos, pero se echa de menos, tanto en el Proyecto de Ley como en lo recomendado por los miembros de la Comisión de Familia, la propuesta de medidas que, a medio plazo, cambien la mentalidad de la población chilena. En la actualidad, pese a la legislación y a sus carencias, los padres chilenos se suelen implicar poco o nada en la crianza de sus hijos. Por lo demás hay que señalar que las medidas propuestas por el Proyecto en análisis afecta a toda persona que tenga 
contrato, dejando fuera del ámbito protector que diseña, una vez más, a las miles de chilenas que son amas de casa o que son asistentas de hogar (o profesionales) y trabajan por horas. Pese a las buenas intenciones de los legisladores, la realidad en Chile es que en el mundo laboral la mujer sufre todo tipo de discriminación y no se han presentado proyectos que apunten a los problemas de fondo (tanto económicos, políticos, sociales, culturales etc.) que de una manera efectiva y completa puedan anular esta injusta situación. Una vez más debemos hacer alusión a las tendencias económicas y sociales imperantes en el parlamento chileno: No existe el menor pudor en mantener políticas económicas que anulan los derechos del trabajador, al tiempo, que en materia axiológica se convierten en guardianes de una moral determinada. De esta manera en entendible, que no justificable, la ausencia de verdaderas políticas de género, pese a que haya medidas que responda a intereses inmediatos (periodos electorales) o a la sensibilidad particular de algunos diputados o senadores, tal y como se ha subrayado anteriormente, que, no obstante, pueden no corresponder a las directrices generales de su partido.

3.1.7. Proyecto de Ley que:’Modifica el artículo 14 de la ley $\mathrm{N}^{\circ} \mathbf{2 0 . 0 6 6}$, sobre violencia intrafamiliar, para facilitar al Ministerio Público el inicio de la investigación del delito de maltrato habitual". Congreso Nacional de Chile. 17 de Julio de 2007. Boletín del Congreso Nacional $N^{\circ}$ 5200-07. Historial: Segundo trámite constitucional. Senado. 20 de Noviembre de 2007

La idea matriz del proyecto que "Modifica el artículo 14 de la Ley $\mathrm{N}^{\circ}$ 20.066, sobre violencia intrafamiliar, para facilitar al Ministerio Público el inicio de la investigación del delito de maltrato habitual" consiste en permitir al Ministerio Público, iniciar la investigación del delito de maltrato habitual, cualquiera fuese la manera en que tomare conocimiento de la eventual ejecución del mismo. En general es muy escasa la participación de los diputados del Partido Demócrata Cristiano y de Renovación Nacional. Si bien se encuentra fuera del objeto de nuestra investigación, no podemos por menos que destacar que son muy abundantes las intervenciones de los diputados del Partido por la Democracia y del Partido Socialista, siempre con un mayor sentido de los problemas de género. Aún así debemos destacar que las campañas que protagoniza el gobierno de la Concertación (de tendencia de centro - izquierda) en contra de la violencia intrafamiliar no suele mantener una postura de género estricta pues bajo tal concepto se incluye todo tipo de agresiones realizadas a los integrantes de una familia (24). De cualquier manera podemos constatar que la prensa se muestra receptiva frente al grave problema de la agresión intrafamiliar, sobre todo a partir del inicio del nuevo milenio, constatando que se trata de un problema que afecta a la calidad de vida de todo el país, tal y como explica Adriana Delpiano, ministra del SERNAM (25)

3.1.8. Proyecto de Ley que "Modifica el Código Penal, para incorporar como agravante en el delito de homicidio, la circunstancia de embarazo de la víctima y que, como consecuencia del hecho punible, se extinga la vida del hijo nonato". Congreso Nacional de Chile, 30 de Septiembre de 2008. Boletín del Congreso 
Nacional $N^{\circ}$ 6109-07. Historial: Primer trámite constitucional. Sin urgencia. 30 de Septiembre de 2008.

La Constitución Política de Chile establece en el artículo 19 número 1, inciso segundo que: "la ley protege la vida del que está por nacer". No obstante, los autores de la moción que "Modifica el Código Penal, para incorporar como agravante en el delito de homicidio, la circunstancia de embarazo de la víctima y que, como consecuencia del hecho punible, se extinga la vida del hijo nonato" consideran que en caso de que una mujer sea asesinada, la condena por la muerte del nonato no está suficientemente penada (Presidio menor en sus grados mínimo y medio). La moción ha sido propuesta recientemente por lo que tan solo ha tenido lugar su presentación y su próximo paso es la presentación en la Comisión de Constitución, Legislación y Justicia. Pese a todo pueden hacerse una serie de comentarios a nuestro juicio, muy importantes y esclarecedores: La defensa del "nasciturus" es una de los temas que muestran un mayor quiebre entre la opinión pública y las autoridades parlamentarias y este proyecto es una clara muestra de política de género regresiva o discriminatoria de la mujer. Nos parece plausible la defensa del que está por nacer, si bien el Pacto Internacional de Derechos Económicos, Sociales y Culturales defiende que ninguna mujer debe poner en peligro su vida por causa del embarazo. Llama, por otra parte la atención que, en caso de asesinato de la mujer embarazada muestren los legisladores una extrema preocupación por la vida del "nasciturus", pero no rebelen preocupación alguna por las embarazadas que, debido al sistema económico imperante, se ven sometidas a trabajos peligrosos que pueden provocarles abortos espontáneos. Pese a las paradojas señaladas los legisladores no reaccionan en su mayoría. La mujer se encuentra desamparada toda vez que se enfrenta a una situación económica - social perversa, la distribución de la píldora está prohibida por decisión del Tribunal Constitucional, las opciones sexuales que difieren de la tradicional son vistas con prejuicio por la sociedad, la paternidad responsable es, con frecuencia, incompatible con ideologías conservadoras, son numerosas las agresiones sexuales, etc.

\subsection{Derechos laborales de las mujeres}

\subsubsection{Proyecto de Ley que "Modifica el Código del Trabajo resguardando el derecho a la igualdad en las remuneraciones", Congreso Nacional de Chile, 19 de Julio de 2006. Boletín del Congreso Nacional $N^{\circ} 4356$ - 13. Historial de la Ley: Ley $\mathbf{N}^{\circ}$ 20.348. Decreto. Oficial. 19 de Junio de 2009.}

La búsqueda de la "igualdad salarial" para "trabajos de igual valor" efectuados por mujeres y hombres es un viejo objetivo de la sociedad internacional una y otra vez frustrado tanto a escala mundial como en el marco de los diferentes países, incluso algunos avanzados en el desarrollo social. Si es cierto que en muchas naciones la legislación existente al respecto se incumple de modo sistemático en Chile era esta una asignatura pendiente incluso en el sistema normativo. De aquí que convenios internacionales ratificados, la discriminación de las trabajadoras y la presión de muchas organizaciones nacionales hacían urgente que se legislara para resolver la "brecha salarial" de género. En efecto el artículo 7, a, 1 del Pacto Internacional de los Derechos 
Sociales, Económicos y Culturales establece el compromiso de los Estados Partes en dicho Pacto para asegurar "un salario equitativo para trabajos de igual valor; sin distinciones de ninguna especie" en particular dice textualmente el artículo citado "debe asegurarse a las mujeres condiciones de trabajo no inferiores a las de los hombres, con salario igual por trabajo igual". La base de la moción que "Modifica el Código del Trabajo resguardando el derecho a la igualdad en las remuneraciones" se encuentra en la discriminación que sufre la mujer en el trabajo, pese al apoyo de la normativa nacional (Constitución y Código del Trabajo) e internacional. Por lo tanto surge esta moción que pretende explicitar el idéntico derecho de hombres y mujeres con el fin de paliar la injusta situación. Desde el 2006 el proyecto se encontraba estancado en el Senado hasta que la Presidenta Michelle Bachelet mostró su preocupación por el proyecto (26) consiguiendo que la Cámara Alta pase a tercer trámite y se acerque a ser aceptado como Ley de la República (27). Hasta que el 20 de mayo de 2009 el proyecto alcanza rango de Ley, pues la Cámara aprobó los cambios introducidos por el Senado estableciéndose el derecho a la igualdad de remuneraciones entre hombres y mujeres que presten un mismo trabajo (28). Salvo en el discurso de algunos parlamentarios, como el de la diputada Carolina Goic, no se ve una clara intención de erradicar las verdaderas causas de discriminación de la mujer y prevalece la mentalidad conservadora y machista que infravalora el trabajo femenino y no reconoce su adecuación más que en trabajos domésticos no remunerados. No es extraño que ante este panorama hace años (aunque con inquietante actualidad) se alzara la voz de Teresa Valdés, socióloga de la FLACSO destacando que "Si no hubiera existido una red de organizaciones feministas generando conocimiento, haciendo diagnósticos, la Concertación no hubiera hecho nada de lo que hizo". Porque tanto en la creación de SERNAM, como en la Ley de filiación, pasando por la Ley de Violencia Intrafamiliar, las feministas chilenas tuvieron mucho que ver" (29). La discriminación de las mujeres asegura igualmente una mano de obra barata para un conjunto de actividades y formas de relación laboral necesarias para el funcionamiento de la economía global representada en la manufactura y agricultura de exportación así como en los servicios de apoyos a las empresas internacionales. Su menor participación en el mercado de trabajo la lleva a depender de su esposo o compañero por lo que, en situaciones de separación o divorcio, su calidad de vida se puede reducir hasta la pobreza o la indigencia pues queda al arbitrio de las decisiones económicas que su ex pareja disponga para ella y sus hijos. Cabe añadir que durante el matrimonio, a menos que la mujer opte por la separación de bienes, queda a merced de su compañero respecto a las decisiones económicas.

\subsection{Derecho a la educación}

3.3.1. Proyecto de Ley "Protección de la madre escolar o estudiante embarazada", Congreso Nacional de Chile, 5 de julio de 1994. $N^{\circ}$ Boletín 1251-18. Historial de la Ley: Ley de la República $\mathrm{N}^{\circ}$ 19.688. 5 de agosto de 2000.

Nos hallamos ante una iniciativa, claramente democratacristiana, que suscitó numerosas intervenciones de diputados de diferentes partidos. En su tramitación legislativa, el 
Proyecto de Ley pasó por la Cámara de Diputados y por el Senado y la Baja y llegó hasta el tercer trámite. Por sus limitaciones y con fines de mejora fue objeto del veto presidencial y el veto del ejecutivo que supusieron diferentes concreciones antes de ser aprobado en la Cámara Baja y ser aprobado como Ley de la República el 5 de agosto de 2000. Estamos ante una respuesta a la problemática general planteada por la crecida del número de adolescentes embarazadas en colegios conservadores, los cuales reaccionaron tratando de expulsarlas a fin de evitar que se divulgara la presencia en sus centros de niñas consideradas por dichas entidades como moralmente reprobables por su conducta. Esta operación de exclusión se apoyó en el supuesto de que la libertad se enseñanza permitía a los centros defender su ideario. Cuando los legisladores intentaron obligarlos a respetar el derecho de las jóvenes madres a proseguir su educación, los portavoces de los mismos alegaron "colisión de derechos". Los diputados democratacristianos se negaron a aceptar tal colisión y priorizaron el derecho a la educación de las adolescentes embarazadas frente al derecho a la libertad de enseñanza (que en Chile tiende a entenderse como la creación de Centros y la imposición de un ideario determinado en el mismo) así como el derecho de las adolescentes a permanecer en el Centro aún si su conducta privada hubiera contravenido el ideario del centro. Se hace mención en las intervenciones de los parlamentarios al "derecho a la vida del nasciturus", aspecto que a primera vista pudiera parecer no relacionado con el derecho de la adolescente embarazada a permanecer en su centro educativo. Hay que subrayar que es traído a colación en virtud de que se entiende que el conocimiento por los adolescentes de que serían ser expulsados en caso de embarazo podía conducirles al aborto; así los democratacristianos entremezclan argumentos antiabortistas y esgrimen un "derecho del nasciturus" que no se estimaría en otros países, con la defensa de los derechos educativos de las madres solteras. En el curso del debate derecha e izquierda, unidos en la defensa del proyecto, se desvincularon en cuanto al argumento legitimador: La democracia cristiana llegó a hacer del debate ocasión para el desarrollo de una campaña antiabortista y la izquierda lo aprovechó para hacer una defensa del derecho a las libertades sexuales y al propio cuerpo, las políticas públicas de educación sexual, y el respeto a los Derechos Humanos de las mujeres, en particular el de no ser discriminada.

Desde los años ochenta del siglo XX tanto los Organismos Internacionales como la propia sociedad chilena demandaban un cambio cambios de conducta en lo que se refiere a la discriminación de la mujer en general y de la adolescente embarazada en particular. Los movimientos feministas lograron que la sociedad internacional hiciera eco de sus nuevas demandas de libertad sexual y reproductiva que iban notoriamente más lejos que lo que, por las mismas fechas, proponían los parlamentarios pues demandaban el derecho a regular su fertilidad, su maternidad y a disponer de su propio cuerpo. La protección de los derechos sociales y económicos de las mujeres aparece como necesaria para el ejercicio de los Derechos Sexuales y Reproductivos, pues están estrechamente relacionados con el "derecho a la salud", a la asistencia o seguridad social etc. 


\subsection{Derecho a la salud}

3.4.1. Proyecto de Ley que: "Elimina la diferenciación según sexo en la estructura de las tablas de factores que determinan los precios de los planes de salud". Congreso Nacional de Chile. Martes 12 de Agosto de 2008, $\mathbf{N}^{\circ}$ Boletín del Congreso Nacional $\mathbf{N}^{\circ}$ 6030-11. Historial: Primer trámite constitucional. Sin urgencia. 12 de Agosto de 2008

En la presentación del proyecto que" Elimina la diferenciación según sexo en las estructura de las tablas de factores que determinan los precios de los planes de salud" se afirma que en los planes de salud ofrecidos por las ISAPRES se encuentran casos en que las mujeres en edad fértil pagan hasta cuatro veces más que un hombre por el mismo plan contraviniendo derechos consagrados en la Constitución vigente, en el Código del Trabajo y en los Acuerdos Internacionales. Se reconoce que es injusto que los gastos de un embarazo deban ser asumidos exclusivamente por la mujer y por lo tanto las responsabilidades económicas del parto, exámenes, cuidados del recién nacido en caso necesario deben ser compartidos por hombre y mujer dado que ambos han participado en la procreación del nuevo ser. Pero no se señala la responsabilidad que tiene el Estado, en aras de conseguir la dignidad de la mujer a través de políticas que motiven el cambio de mentalidad para alcanzar la igualdad del hombre y de la mujer. Se subraya, a continuación que la tasa de natalidad (baja en Chile) debe ser revertida y que la discriminación de la mujer respecto al pago de los planes de las ISAPRES no contribuye a ello. A nuestro entender, independientemente de la tasa de natalidad o de que las mujeres tengan más o menos hijos, no deben ser discriminadas imponiéndoseles planes más caros que los de los varones y con menos prestaciones. El respeto de la mujer, de su dignidad y la no vulneración de los Derechos Sexuales y Reproductivos de las mismas debieran ser el verdadero objetivo de este proyecto que, pese a lo que pudiera pensarse no hace gala de un verdadero espíritu de concepto de género emancipador sino que responde a ideologías tradicionales tendentes a promocionar políticas pro natalistas. Es necesario destacar la enorme voluntad que la ex- presidenta Michelle Bachelet ha demostrado en revertir la vulneración de los derechos de la mujer tal y como atestigua su agenda social en la que puede destacarse la rebaja de 65 a 60 años para acceder gratuitamente a la salud en el sistema público, se otorga atención integral a las víctimas de violencia familiar (con lo que se considera a la violencia intrafamiliar como un problema de salud pública), se permite a los convivientes ser carga de sus respectivas parejas y por primera vez los hombres podrán ser carga de su mujer. Aparece pues en la mandataria una clara conciencia de género reivindicativa. En definitiva, podemos afirmar que un mal sistema de salud provoca empobrecimiento en todos los campos, no sólo en el médico, pues la salud cumple un papel redistributivo que corrige inequidades, consiguiendo una enorme mejora de la calidad de vida de la mujer toda vez que es la gran perjudicada por el actual sistema de salud de Chile. En la actualidad la mayor esperanza de vida de la mujer respecto al hombre redunda en su perjuicio al necesitar en su vejez más cuidados médicos y medicinas, que obtendrá según su capacidad económica. 


\section{CONCLUSIÓN}

Las fuentes legislativas se muestran como indispensables en lo que respecta al estudio de la situación de la mujer en Chile. A través de las fuentes legislativas podemos exponer claramente la interrelación que existe en Chile entre los diferentes actores ya sean los parlamentarios, el público en general, poderes fácticos como las diferentes iglesias (muy influyentes en el país andino), los medios de comunicación (generalmente dominados por corrientes conservadoras en lo que respecta a los aspectos axiológicas pero de un carácter extremadamente liberal en lo económico y que supone una de las grandes paradojas de la élite política, económica y social chilena) etc....

No podemos dejar de mencionar a las numerosas organizaciones feministas sin cuya labor, la situación de la mujer en Chile sería aún más precaria de lo que aún es en la actualidad.

Estas asociaciones realizan un esfuerzo enorme por atenuar las estructuras patriarcales machistas y misóginas que condenan a la mujer a una situación de segundo plano, respecto al varón, en las sociedades latinoamericanas en general y chilena en particular.

A través de protestas, asambleas, ponencias y actos públicos buscan concienciar a la población sobre las vulneraciones de los derechos de más de la mitad de los habitantes de Chile.

De esta manera observamos con nitidez como los diferentes actores son causa y consecuencia, al tiempo, de la evolución que ha sufrido en las últimas décadas la situación de la mujer.

Los actores conservadores ya expuestos, no pueden por menos que tener en cuenta a los interlocutores mas progresistas como son la misma población chilena y las organizaciones feministas, así como el discurso internacional de los Derechos Humanos, si bien es cierto que es necesario subrayar que el concepto de progresismo se encuentra muy diluido en comparación con Europa en lo que respecta a los derechos políticos, económicos, sociales y sexuales de las mujeres

De la misma manera, a través de las propuestas legislativas podemos concluir que es mucho el camino que queda por andar a la mujer chilena en pro de la consecución de todos sus derechos.

\section{BIBLIOGRAFÍA}

ARA PINILLA, Ignacio, "La semántica de los Derechos Humanos" en Anuario de Derechos Humanos, Madrid, Instituto de Derechos Humanos, $\mathrm{N}^{\mathrm{o}} 6$, 1990, pp. 23 y ss.

ARA PINILLA, Ignacio: Las transformaciones de los Derechos Humanos. Madrid. Tecnos. 1990.

LÓPEZ CALERA, Nicolás María, Introducción a los Derechos Humanos, Granada Ed. Comares, 2000; 
NIKKEN, Pedro, La protección internacional de los Derechos Humanos: su desarrollo progresivo, Madrid, Instituto Interamericano de Derechos Humanos-Editorial Civitas, 1987.

Consejo General del Poder Judicial, "Consolidación de derechos y garantías: Los grandes retos de los Derechos Humanos en el siglo XXI" Madrid, Seminario conmemorativo del 50 aniversario de la Declaración Universal de los Derechos Humanos Consejo General del Poder Judicial, 1999.

ATIENZA, M., "Derechos naturales o Derechos Humanos: un problema semántico" en AA.VV, Política y Derechos Humanos, Valencia, Fernando Torres, pp. 17 y ss.

BULYGIN, E.: "Sobre el status ontológico de los Derechos Humanos" en Doxa, No 4, Alicante, 1987; Fioravanti, M. Los derechos fundamentales. Madrid, Trotta, 1996; Laporta, F.: "Sobre el concepto de Derechos Humanos" en Doxa, № 4, Alicante, 1987.

PÉREZ LUÑO, Enrique: "Concepto y concepción de los Derechos Humanos" en la Revista Doxa. N. ' 4.1987; Ibid, "Delimitación conceptual de los Derechos Humanos" en Derechos Humanos, Estado de Derecho y Constitución, Madrid, Tecnos, 1984, pp. 21-51;

ROBLES, Gregorio, Los derechos fundamentales y la ética en la sociedad actual, Madrid, Ed. Cívitas, 1992.; RODRÍGUEZ PANIAGUA, J. Ma: "Los Derechos Humanos como obligación" en Persona y Derecho, Vol., 22, 1990, pp. 234 y ss. ;

VARGAS VALENCIA, Aurelia (compiladora) Derechos Humanos Filosofia y Naturaleza, México, UNAM, 2000; VASAK, K, Les dimensions universelles des droits de l'homme. Bruselas, Bruillant.1990.

PÉREZ LUÑO, A, E (1993 y 1995); Beuchot O. P., M, (1995); Peces-Barba Martínez, G (1998); Pérez Luño, A, Concepto y concepción de los Derechos Humanos (acotaciones a la Ponencia de Francisco Laporta). Biblioteca virtual Miguel de Cervantes. n4 1987; Alexy, R, (1993);Massini, Carlos Ignacio (1987); Bockenforde, Ernst-Wolfgang, (1994);

PÉREZ LUÑO, A. E. (1984); Rodríguez Toubes Muñiz.” R. Opus cit; Hervada, Javier (editor), Juan Pablo II y los Derechos Humanos, Pamplona, EUNSA, 1982.

DELGADO PINTO, José: De nuevo sobre el problema del Derecho Natural. Salamanca. Eds. Universidad. 1982).

RUÍZ MIGUEL, A. (1990), pp. 149 y ss.

Maturana, H y Varela, F, El árbol del conocimiento, Editorial Lumen Humanitas, Buenos Aires, 2004.

Organización Internacional del Trabajo, [http://www.ilo.org].27/02/2009.

SQUELLA NARDUCCI, A.: "Democracia y Derechos Humanos" en Anuario de Derechos Humanos, $\mathrm{N}^{\circ} 7$, Instituto de Derechos Humanos, Facultad de Derecho de la Universidad complutense de Madrid, Madrid, 1990, pp.221 y ss.

ABRAMOVICH,Víctor y Curtis Christian, Los derechos sociales como derechos exigibles. Madrid, Trotta, 2006.

CONTRERAS PELÁEZ. Francisco J Derechos sociales: Teoría e ideología, Madrid, Tecnos, 1994. 
JIMÉNEZ, Eduardo Pablo, Los Derechos Humanos del la Tercera Generación, Buenos Aires, EDIAR, 1997

GONZÁLEZ, G, (coord.), Derechos Humanos: La condición humana en la sociedad tecnológica, Madrid, Tecnos, 1999; Joule, D, Ética del desarrollo. Madrid, IEPALA, 1999; Halpern, Pablo, Los Nuevos Chilenos y la batalla por sus preferencias. Santiago, Chile: Editorial Planeta, 2002; Jonas, H, El principio de responsabilidad. Ensayo de una ética para la civilización tecnológica, Barcelona, Herder, 1995.

AMORÓS, Celia, 1994; Añón, Mª J, 2001; Carbajo Vázquez, Judith, 2004;;; González Cortés, M. T. y Gaudó, C. (eds), 2002; Elósegui, M., 2003; Fernández Ruiz-Gálvez, Encarnación, 2003; Figueruelo, Ángela y otras, 2005; García Inda, Andrés y Lombardo, Enmanuela, 2002.; González Bustos María Ángeles, 2006; Ibáñez, Ma L., 2004; Maquieira; Virginia (coord.),2006; Nash; Nash, Mary, 2004; Rodríguez Piñeiro, M, 2001;; Young, I. M, 2000

AllamAND. Andrés.). El Desalojo. Por qué la Concertación debe irse el 2010 .Santiago de Chile. Aguilar. 2007. p 104.

Informe Sombra al CEDAW. 2003 - 2006.

Superintendencia de la Salud. [ www.supersalud.cl ]. 23 de abril de 2009.

BOSCH, Esperanza et allí, El laberinto patriarcal. Reflexiones teórico-prácticas sobre la violencia sobre las mujeres, Barcelona, Anthropos Editorial, 2006; Fontana, Beatriz, De vergüenzas y secretos. Consideraciones sobre la violencia sexual en la pareja, Espacio Editorial, Buenos Aires - Argentina, Noviembre, 2004; Garrido, Vicente, Amores que matan: acoso y violencia contra las mujeres, Alzira, Algar, 2001. Osborne, Raquel, La violencia contra las mujeres, (comp.), Madrid, Universidad Nacional de Educación a Distancia (UNED), Colección Varia, 2001.

Servicio Nacional de la Mujer (SERNAM). 2010.

SARAMAGO, J, "Palabras del premio Nobel de Literatura 1998", Memorias del encuentro de Intelectuales y Artistas en defensa de la humanidad, Caracas, Venezuela, Fundación Editorial, 2004, p 18.

El Mercurio. 29 de Abril de 2009.

Archivo del Congreso. Primer Trámite Constitucional. Cámara de Diputados. 7 de Diciembre de 2006.

Archivo del Congreso. Primer Trámite Constitucional. Cámara de Diputados. 3 de Abril de 2007.

La Nación. 20 de julio de 2007.

El Mercurio. 23 de julio de 2002.

El Mercurio. 6 de Agosto de 2001

El Mercurio. 10 de Noviembre de 2008.

El Mercurio. 13 de mayo del 2009

La Nación. 21 de mayo de 2009.

El Mercurio. 14 de Mayo de 2000. 